\title{
RENDIMENTO DE MOTOR ELÉTRICO COMO PARÂMETRO DE DIMENSIONAMENTO DE BITOLA DE ALIMENTADOR CONECTADO DIRETAMENTE AO TRANSFORMADOR
}

\section{DELLY OLIVEIRA FILHO ${ }^{1}$, CARLOS A. TEIXEIRA ${ }^{2}$, ADÍLIO F. LACERDA FILHO ${ }^{3}$, JOSÉ H. MARTINS ${ }^{4}$, JOSUÉ M. QUEIROZ ${ }^{5}$}

\begin{abstract}
RESUMO: O dimensionamento de condutores de energia elétrica não leva em consideração critérios de racionalização do uso de energia elétrica. $\mathrm{O}$ estudo abrangeu uma avaliação sobre o dimensionamento de condutores alimentadores de energia elétrica de motores conectados diretamente a transformadores utilizados na eletrificação rural. No dimensionamento dos condutores alimentadores, indica-se que, além do atendimento à norma técnica (dimensionamento em função da capacidade de corrente do condutor e da queda de tensão admissível), também sejam considerados: (i) o número de horas de funcionamento; (ii) as características elétricas e preço de condutores; (iii) as características da instalação, como comprimento do ramal e potência do motor. Provou-se que, em algumas situações, é vantajoso aumentar a bitola do condutor além daquela exigida pela norma técnica, a fim de se economizarem energia e gastos pela menor perda no alimentador e maior rendimento do motor elétrico.
\end{abstract}

PALAVRAS-CHAVE: motor elétrico, qualidade da energia, dimensionamento de condutores.

\section{ELECTRIC MOTOR EFFICIENCY AS PARAMETER FOR SIZING A DIRECTLY CONNECTED INTO TRANSFORMER FEEDER CABLE}

\begin{abstract}
The feeder conductors sizing does not take into account criterion for rational use of electricity. This study is about feeder conductors sizing evaluation for motors connected directly to transformers used in rural electrification. In the feeder conductors sizing indicate that besides the attendance of the technical standard (i.e. sizing as a function of the feeder current capacity and the allowable voltage drop) is also to be considered: (i) the number of working hours, (ii) the feeder's electrical characteristic and price, (iii) the installation characteristics as length and engine's rate. According to the above, in some situations it may be advantageous to increase the gauge of the conductor. It was proven that in some situations it is advantageous to increase the conductor gauge beyond that required by the standards in order to save energy and expenses by the lower feeder losses and by the electric motor higher efficiency.
\end{abstract}

KEYWORDS: electric motor, energy quality, conductors sizing, rural electrification.

\section{INTRODUÇÃO}

Com o aumento da automatização dos processos agrícolas, a demanda de energia elétrica para o setor agropecuário tem aumentado significativamente. A Figura 1 apresenta o aumento do consumo de eletricidade para os principais setores da economia.

O Balanço Energético Nacional de 2009 (ano base 2008) informa que o setor agropecuário consome $4 \%$ da energia elétrica do País. Não se pode precisar o percentual desta energia destinada à

\footnotetext{
${ }^{1}$ Professor, Departamento de Engenharia Agrícola, Universidade Federal de Viçosa, Viçosa MG, delly@ufv.br.

${ }^{2}$ Professor, Departamento de Agronomia, Universidade Federal Rural de Pernambuco, Serra Talhada PE, carlos.teixeira@uast.ufrpe.br.

${ }^{3}$ Professor, Departamento de Engenharia Agrícola, Universidade Federal de Viçosa, Viçosa MG, alacerda@ufv.br.

${ }^{4}$ Professor, Departamento de Engenharia Agrícola, Universidade Federal de Viçosa, Viçosa MG, jhmartins@ufv.br.

${ }^{5}$ Estudante de mestrado em Engenharia Agrícola, Universidade Federal de Viçosa, Viçosa MG, josue.queiroz@ufv.br.

Recebido pelo Conselho Editorial em: 16-12-2009

Aprovado pelo Conselho Editorial em: 23-8-2010
} 
utilização de cargas que demandam tração, mas acredita-se que este valor seja significativo, ou seja, pelo menos $50 \%$.

$\mathrm{Na}$ zona rural, a utilização de transformadores monofásicos corresponde a $75 \%$ dos equipamentos instalados e $25 \%$ para transformadores trifásicos. A potência média dos transformadores utilizados na zona rural está em torno de $22 \mathrm{kVA}$ (monofásico e trifásico).

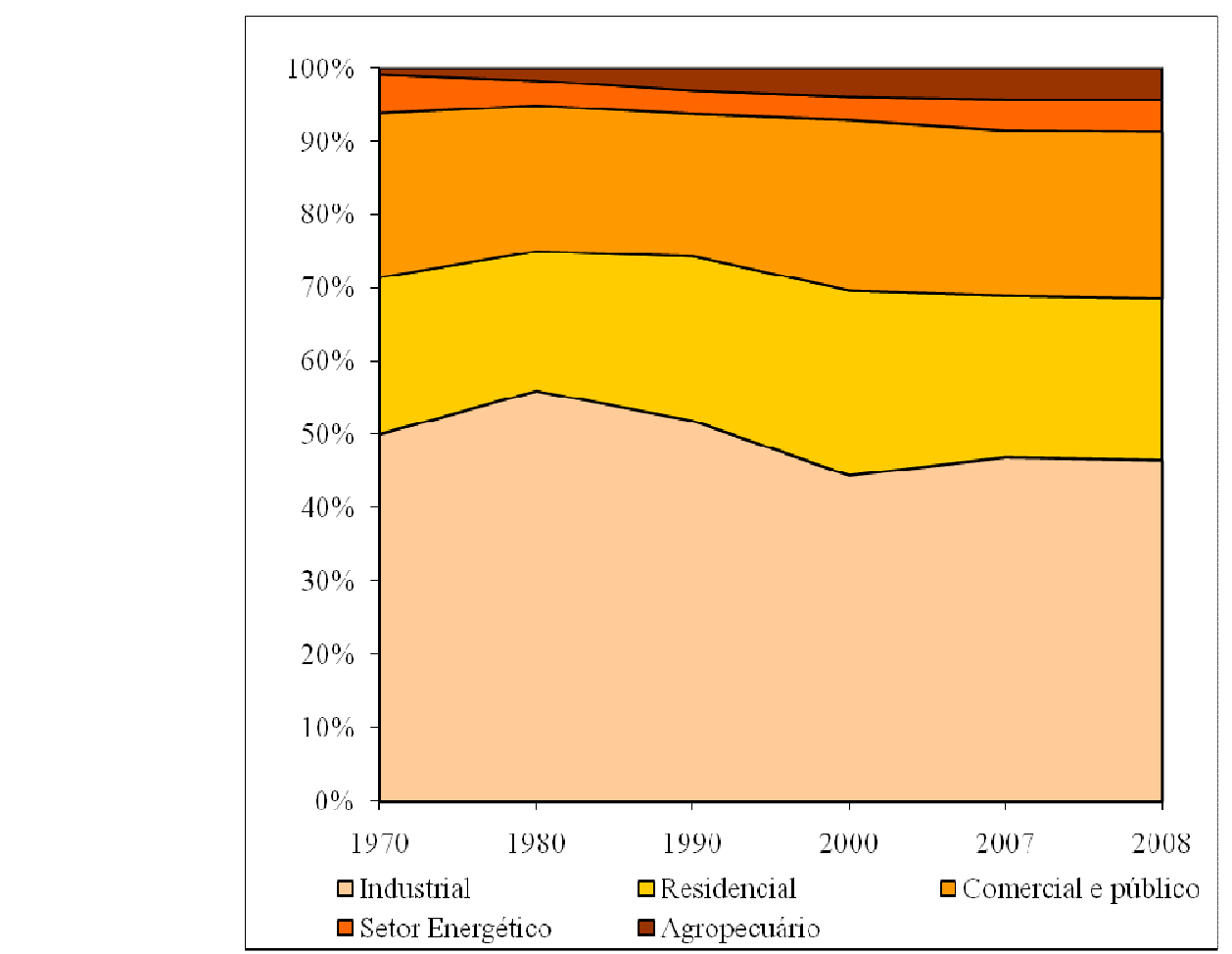

Fonte: (BEM, 2009).

FIGURA 1. Consumo de energia elétrica por setor da economia. Electrical energy consumption by economy sector.

O dimensionamento de cabos alimentadores considera dois critérios: o da queda de tensão admissível e o da corrente máxima. Estes dois critérios devem ser obrigatoriamente obedecidos de forma simultânea. Quando a carga é relativamente distante, o critério de queda de tensão será o mais exigente, portanto prevalecerá no dimensionamento de cabos. Mas quando a carga é relativamente próxima, o critério da corrente máxima admissível prevalecerá.

O arranjo típico de sistemas de eletrificação rural é diferente dos sistemas industriais e, no que diz respeito a dimensionamento de condutores para alimentação de motores elétricos, carece de estudo aprofundado.

Em geral, no setor industrial, a carga final é alimentada por meio de centro de comando/controle de motores que, por sua vez, são alimentados por quadros gerais de distribuição, e estes, pelo transformador de entrada. Assim, quando as cargas são conectadas por meio de centro de distribuição de energia, no caso de nem todas as cargas serem ligadas simultaneamente, os valores de quedas de tensão utilizadas para efeito de cálculo no projeto não serão observados.

Já em muitos casos observados na eletrificação rural, as cargas são alimentadas diretamente pelos transformadores, e as quedas de tensão de projeto podem estar realmente ocorrendo.

Assim, um cálculo de dimensionamento dos cabos leva em conta o fator de carga, ou seja, os motores não são ligados simultaneamente na maioria das aplicações. No setor agropecuário, é comum encontrar configurações onde as cargas (em especial os motores) são alimentadas pelo transformador da propriedade através de proteções adequadas. 
A norma NBR 5410 (ABNT, 2004) permite uma queda de tensão de $7 \%$ a partir do secundário do transformador MT/BT até o ponto de utilização, e a resolução $\mathrm{n}^{\mathrm{o}} 505$, de 26 de novembro de 2001 da ANEEL, aceita que a tensão de fornecimento varie 5\% (para mais ou para menos) da tensão nominal para unidades consumidoras atendidas em tensão superior a $1 \mathrm{kV}$.

Recentemente, tem havido uma crescente preocupação com o uso de energia e seu impacto negativo sobre o meio ambiente. A maioria dos países em desenvolvimento tem cada vez mais industrializado seus processos agrícolas. Introduzido, o conceito de utilização racional de energia visa a reduzir o consumo de energia e também corresponde a uma utilização otimizada de todos os recursos econômicos (SAIDUR, 2009).

BORGES NETO \& CARVALHO (2009) elaboraram um programa computacional para auxílio ao planejamento energético na eletrificação rural, porém o programa não leva em consideração a qualidade de energia no dimensionamento de alimentadores.

\section{Qualidade do fornecimento de energia elétrica}

A qualidade da energia ofertada pela concessionária de energia elétrica pode ser monitorada pelo consumidor de uso final por meio de: desequilíbrio da rede trifásica, com tensões apresentando diferentes amplitudes e/ou defasagens; alterações ou flutuações da amplitude da tensão; e existência de forte conteúdo de harmônicos, alterando o comportamento senoidal da tensão (AUGUSTO JÚNIOR \& CHABU, 2002).

Estes três parâmetros constituem as principais distorções que podem ocorrer nas redes trifásicas, pois sua presença altera o desempenho de todos os equipamentos elétricos, os quais são especificados para determinados níveis de tensão, defasagem entre as fases e índice de distorção harmônica.

Utilizando a ferramenta de cálculo "componentes simétricas", encontra-se o desequilíbrio de uma rede. Em sistema trifásico, estas componentes estão divididas em: sequências positiva, negativa e zero, considerando diferentes amplitudes de tensão e/ou defasagens diferentes de $120^{\circ}$. De acordo com o ELETROBRÁS (2004), segundo a NBR 7094 (ABNT, 2003), um sistema trifásico é considerado praticamente equilibrado se a componente de sequência zero não exceder $1 \%$ da componente de sequência positiva, bem como se a componente de sequência negativa não exceder $1 \%$ da componente de sequência positiva, durante período prolongado, ou $1,5 \%$ durante um período curto não superior a alguns minutos.

O cálculo do desequilíbrio, baseado apenas, na consideração de amplitudes diferentes, pode acobertar erros. Entretanto, como a medição da defasagem exige instrumentação apropriada, a nível industrial, a NBR 7097 (ABNT, 1996) admite, para fins práticos, que o desequilíbrio da rede seja definido por:

$$
\mathbf{D}_{\text {tensão }}=\frac{V_{\max }-V_{\text {média }}}{V_{\text {média }}}
$$

em que,

$\mathrm{D}_{\text {tensão }}$ - desequilíbrio de tensão, \%;

$\mathrm{V}_{\text {máx }}$ - valor da máxima tensão, $\mathrm{V}$, e

$\mathrm{V}_{\text {média }}$ - valor médio das tensões, $\mathrm{V}$.

O efeito do desequilíbrio da tensão nas correntes dos motores elétricos trifásicos pode ser observado na Figura 2.

Estudos demonstraram que um pequeno desequilíbrio de 3,5\% na tensão pode aumentar as perdas do motor de indução trifásico em 20\%. Um desequilíbrio de $5 \%$ ou mais pode destruí-lo, rapidamente. Segundo a norma NBR 7097 (ABNT, 1996), devem ser aplicados fatores de redução para a potência disponível no eixo de motores categoria $\mathrm{N}$, quando os mesmos são alimentados por tensões desequilibradas, de modo que a elevação de temperatura admissível não seja excedida. Para 
as outras categorias, é recomendável consultar o fabricante (ELETROBRÁS, 2004). Para os fatores de redução de potência disponível, o eixo a ser adotado pode ser observado na Figura 3.

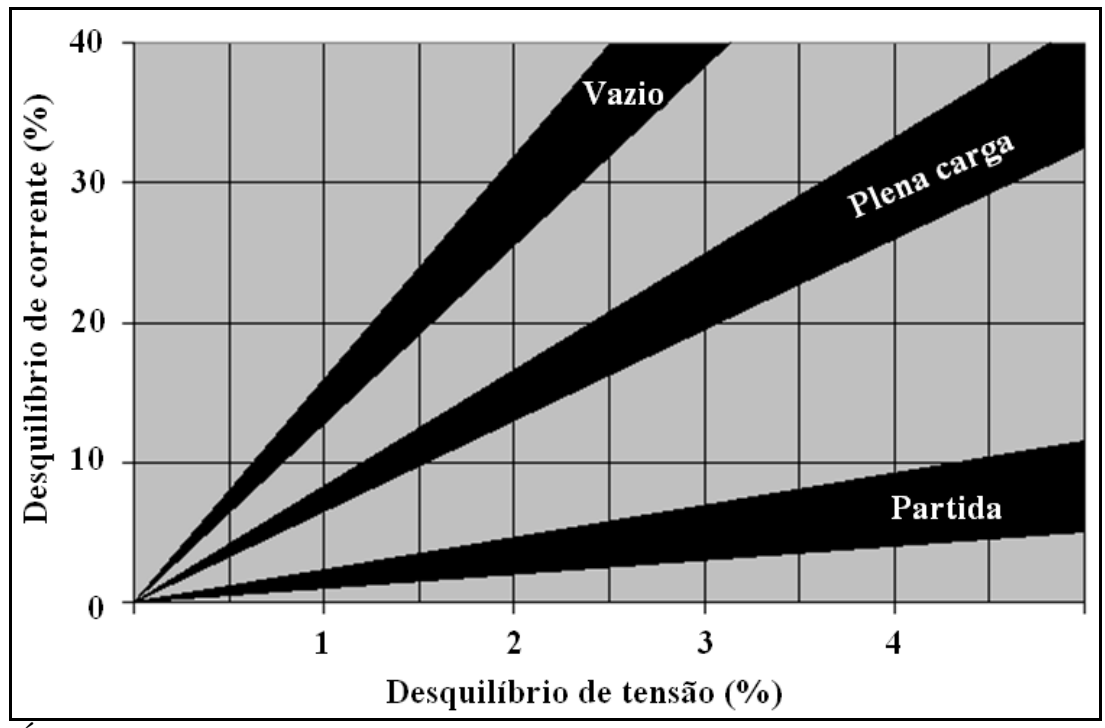

Fonte: ELETROBRÁS (2002).

FIGURA 2. Efeito do desequilíbrio da tensão nas correntes de um motor de indução trifásico. Effect of voltage imbalance in the currents of a three-phase induction motor.

Fonte: ELETROBRÁS (2002).

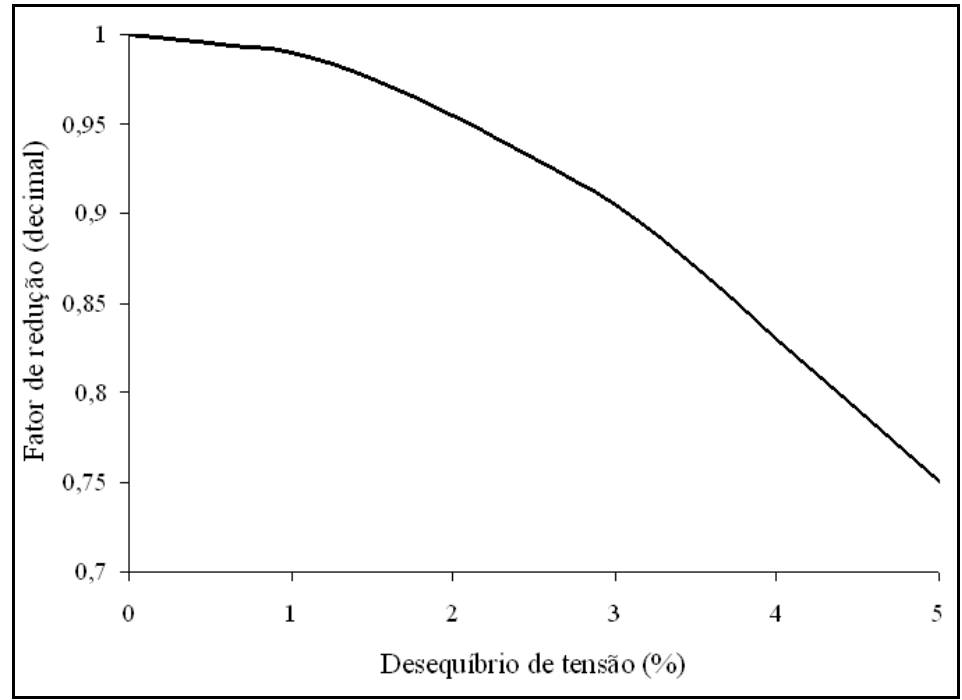

FIGURA 3. Curva do fator de redução para a potência disponível no eixo. Reduction factor Curve of the power available on the shaft.

A alteração na amplitude de tensão pode influenciar na eficiência e no fator de potência de motores elétricos, pois este equipamento é influenciado, diretamente, pelo valor da tensão de alimentação (FAIZ et al., 2006). Normalmente, estes motores são projetados para suportar variações máximas numa faixa de $10 \%$ acima e abaixo da tensão nominal. Na Figura 4, apresenta-se a influência da variação da tensão no desempenho do motor de indução trifásico. 


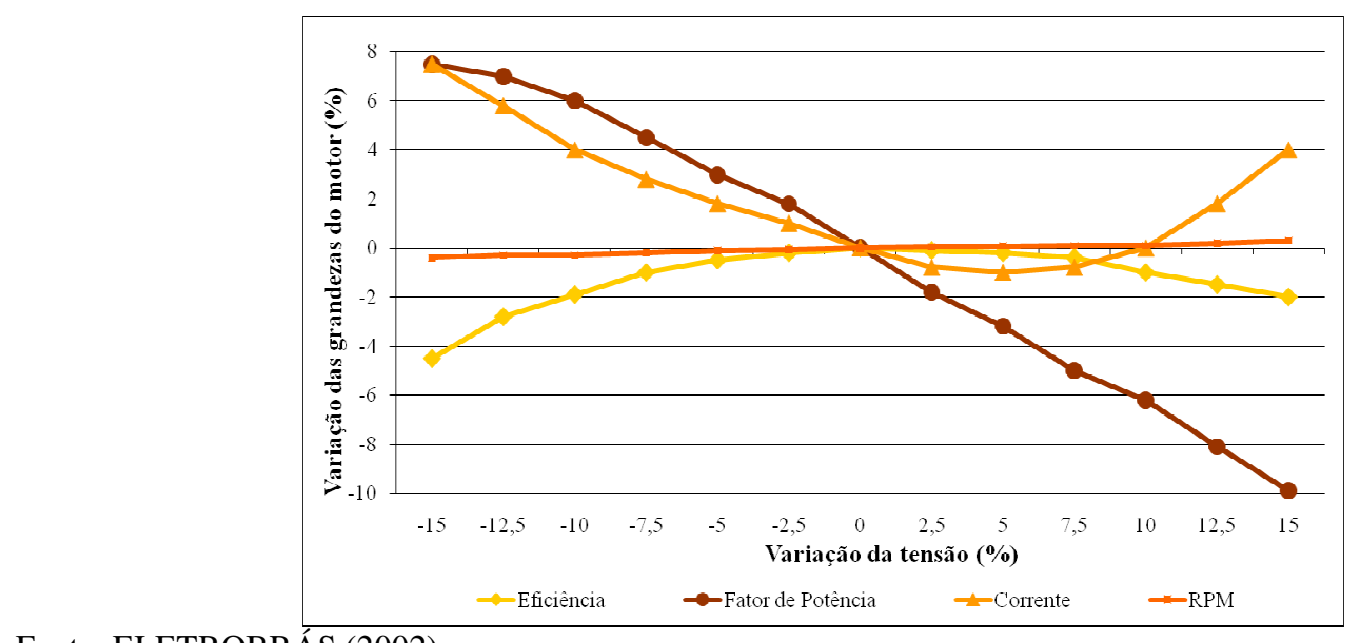

Fonte: ELETROBRẤS (2002).

FIGURA 4. Influência da variação da tensão no desempenho de um motor de indução trifásico. Influence of the voltage variation in the performance of a three-phase induction motor.

\section{Análise do dimensionamento de condutores para motores elétricos considerando a relação entre o aumento da seção do condutor e a queda de tensão admissível}

Segundo CREDER (2007), a norma técnica permite que haja uma queda de tensão máxima de $7 \%$ nos condutores alimentadores, desde o quadro geral de distribuição até o motor elétrico. De acordo com a ELETROBRÁS (2002), há a influência da variação da tensão no desempenho de um motor de indução trifásico, principalmente no rendimento do motor elétrico. Para uma queda de $7 \%$ na tensão, há uma redução de cerca de $1 \%$ no rendimento de um motor elétrico de indução trifásico (Figura 4). Uma possibilidade para minimizar a perda de rendimento dos motores elétricos é o estudo da relação entre: (i) aumento da bitola do condutor, uma bitola acima, indicada pelo dimensionamento tradicional; (ii) custo entre os condutores de bitolas diferentes; (iii) comprimento do condutor; (iv) horas de funcionamento diário do motor elétrico; (v) preço da energia elétrica; (vi) ganho de rendimento, devido à diminuição da queda de tensão pelo aumento da bitola do condutor; (vii) vida útil dos condutores; e (viii) taxa de juros adotada na análise de vida útil.

Para a análise de vida útil, serão utilizados os seguintes indicadores econômicos: (i) Valor Presente Líquido (VPL); (ii) Taxa Interna de Retorno (TIR); (iii) Razão Benefício/Custo (RB/C), e (iv) Tempo de Retorno de Capital (TRC) (LAPPONI, 1996; FRIZZONE \& SILVEIRA, 2000).

O critério do método do Valor Presente Líquido estabelece que, enquanto o valor presente das entradas for maior que o valor presente das saídas, que foi calculado com a taxa de juros $\mathrm{k}$, que mede o custo de capital, o projeto deve ser aceito. Resumindo, sempre que: (i) VPL $>0$, o projeto deve ser aceito; (ii) VPL $=0$, é indiferente aceitar ou não; e (iii) $\mathrm{VPL}<0$, o projeto não deve ser aceito.

O critério do método da Taxa Interna de Retorno estabelece que, enquanto o valor da TIR for maior que o valor do custo de capital k, o projeto deve ser aceito, isto é, sempre que (i) TIR > k, o projeto deve ser aceito; (ii) $\mathrm{TIR}=\mathrm{k}$, é indiferente aceitar ou não; e (iii) $\mathrm{TIR}<\mathrm{k}$, o projeto não deve ser aceito.

A Razão Benefício/Custo é o quociente entre a soma dos benefícios e dos custos, descontada taxa de juros adotada para cada período considerado. O importante é verificar se os benéficos são maiores que os custos.

O critério do método do Tempo de Retorno de Capital estabelece que: (i) TRC > vida útil do projeto, o projeto não deve ser aceito; (ii) $\mathrm{TRC}$ = vida útil do projeto, é indiferente aceitar ou não, e (iii) TRC < vida útil do projeto, o projeto deve ser aceito. 
Neste trabalho, avaliou-se a racionalização do uso de energia elétrica no dimensionamento de condutores alimentadores de energia elétrica, sugerindo-se uma complementação à norma de dimensionamento de condutores alimentadores de energia elétrica.

\section{MATERIAL E MÉTODOS}

O experimento foi realizado no Laboratório de Energia pertencente ao Departamento de Engenharia Agrícola, na Universidade Federal de Viçosa, Viçosa - MG.

Para a simulação, verificou-se em que a situação poderia ser vantajosa aumentando a bitola do condutor para evitar perda de rendimento nos motores elétricos pelo efeito Joule, considerando-se: (i) vida útil dos equipamentos; (ii) potência nominal dos motores elétricos; (iii) rendimento nominal dos motores elétricos; (iv) valor da tarifa de energia elétrica; (v) número de horas de funcionamento anual do motor elétrico; (vi) preço entre condutores de bitolas diferentes; (vii) rendimento entre condutores de bitolas diferentes; (viii) variação de comprimento dos condutores; (ix) preço entre os condutores de energia elétrica; (x) aumento no preço da tarifa de energia elétrica; e (xi) variação no rendimento do motor elétrico pelo aumento da bitola do condutor alimentador.

Com estes parâmetros, fizeram-se simulações de gastos com energia elétrica na vida útil do equipamento, considerando-se o gasto inicial com a compra de equipamentos.

\section{RESULTADOS E DISCUSSÃO}

De acordo com os dados de entrada para a realização da análise econômica de vida útil para condutores de alimentação de motores elétricos trifásicos, apresentados na Tabela 1, pode-se fazer a análise de vida útil, adotando-se os índices econômicos do valor presente líquido (VPL) e a taxa interna de retorno (TIR) para o estudo do dimensionamento de condutores para motores elétricos, considerando a relação entre o aumento da seção do condutor e a queda de tensão admissível.

Os valores de variação no rendimento do motor elétrico pelo aumento da bitola do condutor alimentador considerados foram 1;2; 3 e 4. Esses valores são plausíveis pelo fato de, além de ser admissível pela norma uma queda de tensão de até $7 \%$ (função da forma de instalação), isto é, motor ligado diretamente ao transformador, as concessionárias de energia elétrica poderem fornecer tensão com flutuação dentro de limites aprovados pela norma. O somatório destes dois efeitos pode alcançar uma variação de tensão de cerca de $12 \%$. Segundo a Tabela 1, uma queda de tensão de $12 \%$ pode causar cerca de $3 \%$ de variação no rendimento do motor elétrico.

TABELA 1. Dados para realização da análise econômica de vida útil para condutores de alimentação de motores elétricos trifásicos. Data to perform the economic analysis of shelf life for the feeder conductors of three phase electric motors.

\begin{tabular}{lcc}
\hline Item & Valor & Unidade \\
\hline Vida útil & $20^{*}$ & ano \\
Taxa de juros & $12^{*}$ & $\%$ ao ano \\
Potência nominal dos motores elétricos & 0,$736 ; 7,36$ e 36,8 & $\mathrm{~kW}$ \\
Rendimento nominal dos motores elétricos, respectivamente & 78,$0 ; 89,0$ e 91,7 & $\%$ \\
Valor da tarifa de energia elétrica & 0,6 & $\mathrm{R}^{*} \mathrm{kWh}^{-1}$ \\
Número de horas de funcionamento anual do motor elétrico & $3.960^{*}$ & $\mathrm{~h}$ ano \\
Variação de preço entre condutores de bitolas diferentes & $30^{*}$ & $\%$ \\
Variação de rendimento entre condutores de bitolas diferentes & $1^{*}$ & $\%$ \\
Variação do comprimento dos condutores & de $50^{*}$ a 13.550 & $\mathrm{~m}$ \\
Variação de preço entre os condutores de energia elétrica & $10 ; 20 ; 30^{*} ; 40 ; 50$ e 60 & $\%$ \\
Aumento no preço da tarifa de energia elétrica & $0^{*} ; 5 ; 10 ; 15 ; 20 ; 25$ e 30 & $\%$ \\
Variação no rendimento do motor elétrico pelo aumento da bitola do & $1 * ; 2 ; 3$ e 4 & $\%$ \\
condutor alimentador & &
\end{tabular}

\footnotetext{
*Valores adotados no caso-base.
} 
De posse dos dados de entrada apresentados na Tabela 1, realizaram-se diversas simulações variando o comprimento dos condutores e a potência dos motores, encontrando os limites onde há viabilidade econômica, ou seja, quando a Taxa Interna de Retorno (TIR) é maior que $12 \%$.

Na Figura 5, apresenta-se a região da TIR maior do que o assumido como viável (12\% ao ano) para a variação de preço entre condutores de energia elétrica em função da distância da carga ao transformador para: (a) motores de 1 a $10 \mathrm{cv}$; (b) motores de 10 a $50 \mathrm{cv}$, e (c) motores maiores que $50 \mathrm{cv}$.

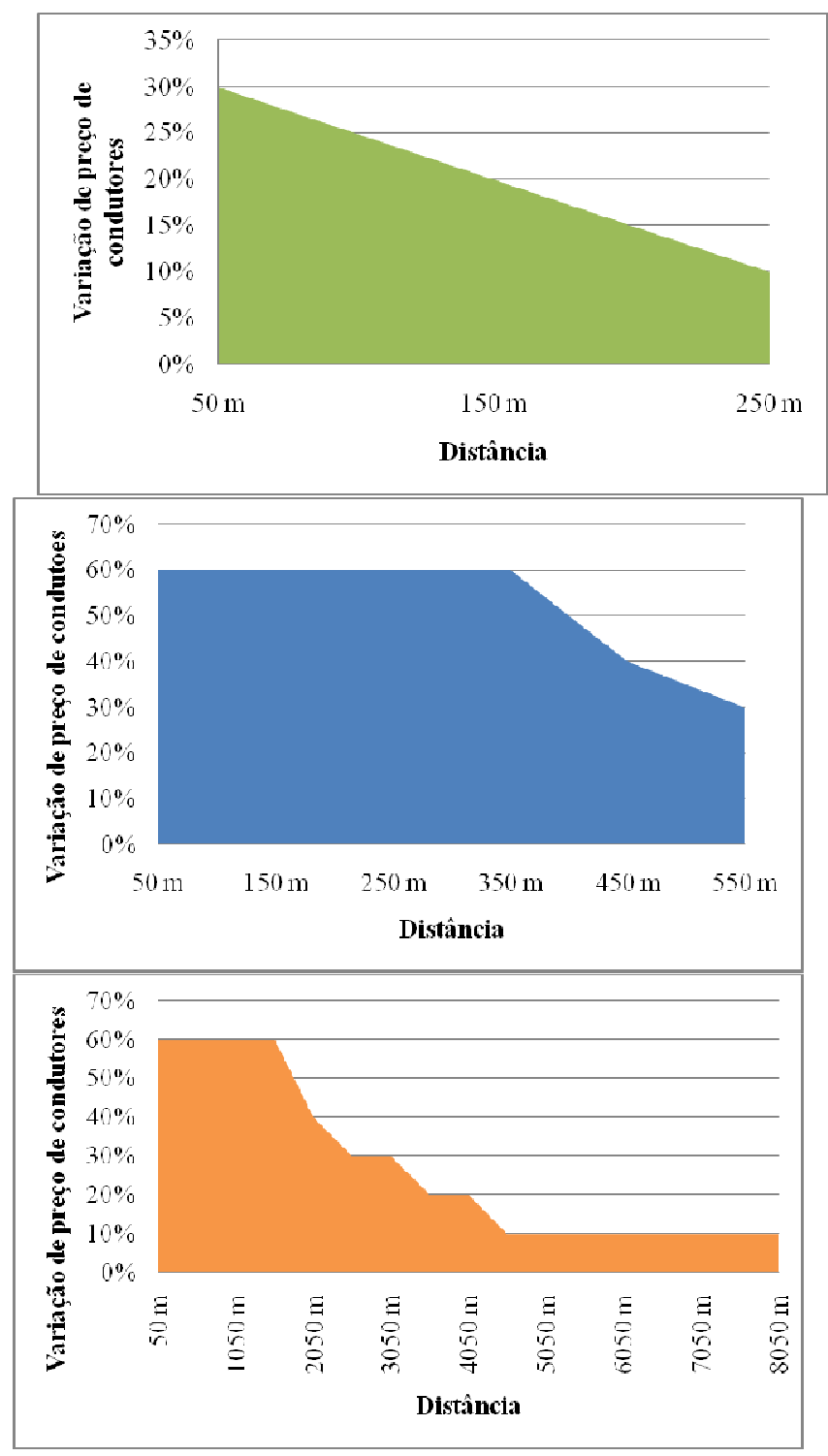

(a)

(b)

FIGURA 5. Região da TIR maior do que o assumido como (viável, $12 \%$ ao ano) para a variação de preço entre condutores de energia elétrica em função da distância da carga ao transformador para: (a) motores de 1 a $10 \mathrm{cv}$; (b) motores de 10 a $50 \mathrm{cv}$, e (c) motores maiores que $50 \mathrm{cv}$. TRI region greater than assumed to be viable, $12 \%$ per year, for the price change between conductors of electricity depending on the distance of the load to the processor to: (a) motors of 1 to $10 \mathrm{hp}$, (b) motors of 10 to $50 \mathrm{hp}$ and (c) motors greater than $50 \mathrm{hp}$.

Conforme Figura 5a, existe viabilidade econômica desta substituição quando: (i) a variação de preço do condutor for menor que $10 \%$, e o comprimento do condutor for menor que $250 \mathrm{~m}$; (ii) a variação de preço do condutor for menor que $20 \%$, e o comprimento do condutor for menor que 
$150 \mathrm{~m}$, e (iii) a variação de preço do condutor for menor que $30 \%$, e o comprimento do condutor for menor que $50 \mathrm{~m}$.

De acordo com a Figura 5b, observa-se que há viabilidade econômica desta substituição quando: (i) a variação de preço do condutor for menor que $30 \%$, e o comprimento do condutor for menor que $550 \mathrm{~m}$; (ii) a variação de preço do condutor for menor que $40 \%$, e o comprimento do condutor for menor que $450 \mathrm{~m}$, e (iii) a variação de preço do condutor for menor que $60 \%$, e o comprimento do condutor for menor que $350 \mathrm{~m}$.

Na Figura 5c, mostra-se que há viabilidade econômica desta substituição quando: (i) a variação de preço do condutor for menor que $10 \%$, e o comprimento do condutor for menor que $8.050 \mathrm{~m}$; (ii) a variação de preço do condutor for menor que $20 \%$, e o comprimento do condutor for menor que $4.050 \mathrm{~m}$; (iii) a variação de preço do condutor for menor que $30 \%$, e o comprimento do condutor for menor que $3.050 \mathrm{~m}$; (iv) a variação de preço do condutor for menor que $40 \%$, e o comprimento do condutor for menor que $2.050 \mathrm{~m}$, e (v) a variação de preço do condutor for menor que $60 \%$, e o comprimento do condutor for menor que $1.150 \mathrm{~m}$.

Continuando com as simulações, variaram-se novamente o comprimento dos condutores e a potência dos motores, mas agora em função da variação do aumento da tarifa de energia elétrica. A Tabela 2 apresenta as distâncias mínimas da carga ao transformador para justificar a inclusão do critério de variação de rendimento de motores no dimensionamento de condutores em função da variação da tarifa de energia elétrica para faixas de potência de motores elétricos.

TABELA 2. Distâncias mínimas da carga ao transformador para justificar a inclusão do critério de variação de rendimento de motores no dimensionamento de condutores em função da variação da tarifa de energia elétrica para faixas de potência de motores elétricos. Minimum distances from the load to the processor to justify the inclusion of the criterion of the engine performance variation in the sizing of conductors as a function of the electricity cost variation for electric motor.

\begin{tabular}{cccc}
\hline \multirow{2}{*}{ Variação da Tarifa de Energia Elétrica } & \multicolumn{3}{c}{ Potência dos Motores (cv) } \\
\cline { 2 - 4 } & 1 a 10 & $10 \mathrm{a} \mathrm{50}$ & $>50$ \\
\hline $5 \%$ & $<150 \mathrm{~m}$ & $<650 \mathrm{~m}$ & $<3.050 \mathrm{~m}$ \\
\cline { 3 - 3 } $20 \%$ & & $<750 \mathrm{~m}$ & $<3.550 \mathrm{~m}$ \\
$30 \%$ & & $<$ \\
\hline
\end{tabular}

De acordo com a Tabela 2, observa-se que há viabilidade econômica da inclusão deste critério quando: (i) para motores de $1 \mathrm{a} 10 \mathrm{cv}$ sempre que a distância for menor que $150 \mathrm{~m}$ para variação nas tarifas de energia elétrica de 5 a 30\%; (ii) para motores de 10 a $50 \mathrm{cv}$, tem-se: para variação nas tarifas de energia elétrica de 5 a $15 \%$, a distância deve ser menor do que $650 \mathrm{~m}$; já para variação nas tarifas de energia elétrica de 20 a $30 \%$, a distância deve ser menor do que $750 \mathrm{~m}$, e (iii) para motores maiores do $50 \mathrm{cv}$, tem-se: para variação nas tarifas de energia elétrica de 5 a $15 \%$, a distância deve ser menor do que $3.050 \mathrm{~m}$; já para variação nas tarifas de energia elétrica de 20 a $30 \%$, a distância deve ser menor do que $3.550 \mathrm{~m}$.

Realizada nova simulação, variaram-se o comprimento dos condutores e a potência dos motores para obter o resultado em função da variação do rendimento pelo aumento da bitola do condutor. Na Figura 6, apresentam-se os resultados.

De acordo com a Figura 6a, observa-se que há viabilidade econômica desta substituição quando: (i) o ganho de rendimento do motor elétrico for igual a $1 \%$ ou $2 \%$, e o comprimento do condutor alimentador for inferior a $150 \mathrm{~m}$; (ii) o ganho de rendimento do motor elétrico for igual a $3 \%$, e o comprimento do condutor alimentador for inferior a $250 \mathrm{~m}$, e (iii) o ganho de rendimento do motor elétrico for igual a 4 , e o comprimento do condutor alimentador for inferior a $350 \mathrm{~m}$. 


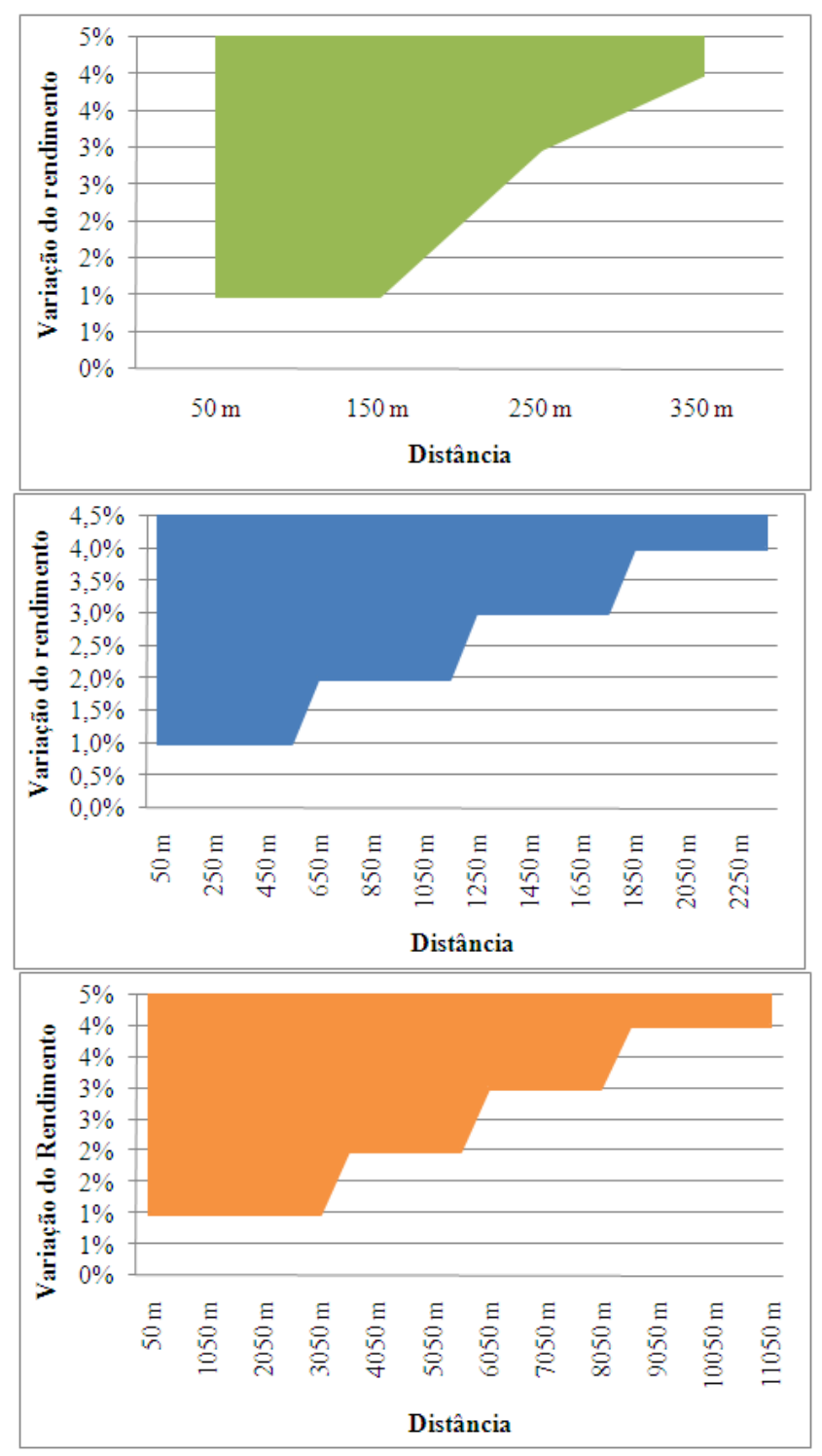

(a)

(b)

(c)

FIGURA 6. Região da TIR maior do que o assumido como viável (12\% ao ano) para a variação do rendimento pelo aumento da bitola do condutor em função da distância da carga ao transformador para: (a) motores de 1 a $10 \mathrm{cv}$; (b) motores de 10 a $50 \mathrm{cv}$, e (c) motores maiores que $50 \mathrm{cv}$. TIR region greater than assumed to be viable, $12 \%$ per year, for the variation in efficiency by increasing the gauge of the conductor as a function of distance from the load to the processor to: (a) motors of 1 to $10 \mathrm{hp}$, (b) motors from 10 to $50 \mathrm{hp}$ and (c) motors greater than $50 \mathrm{hp}$.

Conforme a Figura 6b, observa-se que há viabilidade econômica desta substituição quando: (i) o ganho de rendimento do motor elétrico for igual a $1 \%$, e o comprimento do condutor alimentador for inferior a $550 \mathrm{~m}$; (ii) o ganho de rendimento do motor elétrico for igual a $2 \%$, e o comprimento do condutor alimentador for inferior a $1.150 \mathrm{~m}$; (iii) o ganho de rendimento do motor elétrico for igual a $3 \%$, e o comprimento do condutor alimentador for inferior a $1.750 \mathrm{~m}$; (iv) o ganho de rendimento do motor elétrico for igual a $4 \%$, e o comprimento do condutor alimentador for inferior a $2.350 \mathrm{~m}$.

Na Figura 6c, observa-se que há viabilidade econômica desta substituição quando o ganho de rendimento do motor elétrico for, pelo menos, igual a 1; $2 ; 3$ e 4\%, e o comprimento do alimentador for inferior a $3.050,5.550,8.050 \mathrm{e} 11.050 \mathrm{~m}$, respectivamente. 
Conforme informado na introdução deste artigo, o número de transformadores monofásicos utilizados na zona rural é muito maior que os transformadores trifásicos. E sabe-se que a potência máxima de motores monofásicos é de $12 \mathrm{cv}$. Baseando-se nestas informações, na Figura 7, apresentam-se os resultados/vantagens da simulação para motores entre 1 e $10 \mathrm{cv}$.

De acordo com a Figura 7, pode-se afirma que: (i) para motores distantes $50 \mathrm{~m}$ do transformador e uma variação de preço do condutor de $20 \%$, obtém-se uma TIR de aproximadamente $30 \%$ (Figura 7a); (ii) para aumento da tarifa de energia elétrica menor que 30\% e motor distante $50 \mathrm{~ms}$ do transformador, a TIR varia entre 20 e $25 \%$ (Figura $7 \mathrm{~b}$ ), e (iii) motor distante $50 \mathrm{~m}$ do transformador possui TIR entre 18 e $80 \%$ para variação do rendimento pelo aumento da bitola do condutor entre 1 e $4 \%$ e, no caso de motor distante $150 \mathrm{~m}$, a TIR pode atingir $26 \%$ quando a variação do rendimento pelo aumento da bitola do condutor for 4\% (Figura 7c).

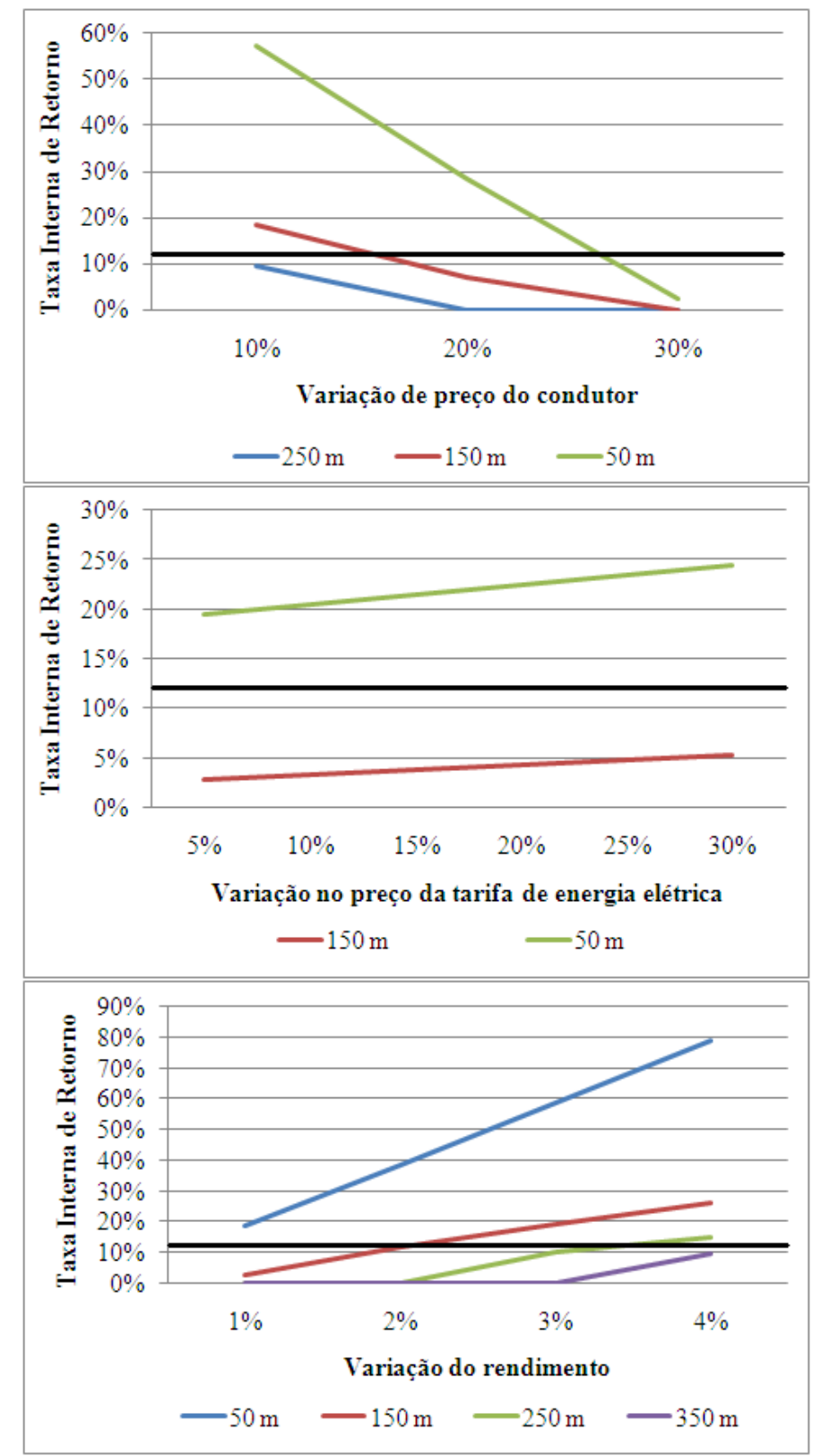

(a)

(b)

(c)

FIGURA 7. Taxa Interna de Retorno da inclusão do critério qualidade de energia em função da variação do: (a) Preço do condutor; (b) Tarifa de energia elétrica, e (c) Rendimento de motores pelo aumento da bitola dos condutores. Internal Rate of Return of the inclusion criteria of power quality due to the variation of: (a) Price of conductor, (b) price of electricity, and (c) efficiency by increasing the gauge of the conductors. 


\section{CONCLUSÕES}

A escolha econômica do condutor para alimentação de motores elétricos deverá atender não somente aos critérios de corrente máxima admissível e queda de tensão, mas também aos seguintes parâmetros: número de horas de funcionamento anual; comprimento do circuito; potência nominal do motor e fator de serviço e custos unitários de tarifa (consumo e demanda) sejam economicamente viáveis, considerando o decréscimo no rendimento de motores elétricos como um fator auxiliar na decisão de aumentar a bitola do condutor alimentador nas três categorias de motores estudadas, havendo situações em que o aumento da bitola dos condutores alimentadores é justificada pela economia de energia elétrica.

Concluí-se também que, no contexto rural, esta análise é ainda mais importante, haja vista que a maioria dos estabelecimentos rurais é eletrificada no sistema monofásico, com diversas cargas conectadas diretamente ao transformador. Então é necessário que seja feita a verificação do dimensionamento de condutores, considerando-se não somente os critérios previstos em norma, máxima queda de tensão e corrente máxima admissível, mas também a variação do rendimento dos motores das características da instalação: custo da energia elétrica, custo de condutores e variação do rendimento dos motores em função da queda de tensão.

\section{AGRADECIMENTOS}

À Coordenadoria de Apoio ao Pessoal de Ensino Superior (CAPES), ao Conselho Nacional de Desenvolvimento Científico e Tecnológico $(\mathrm{CNPq})$ e à Fundação de Amparo à Pesquisa do Estado de Minas Gerais (FAPEMIG).

\section{REFERÊNCIAS}

ABNT. ASSOCIAÇÃO BRASILEIRA DE NORMAS TÉCNICAS. NBR 14039: Instalações elétricas de média tensão de 1,0 kV a 36,2 kV. Rio de Janeiro, 2003.

ABNT. ASSOCIAÇÃO BRASILEIRA DE NORMAS TÉCNICAS. NBR 5410: Instalações elétricas em baixa tensão. Rio de Janeiro, 2004.

ABNT. ASSOCIAÇÃO BRASILEIRA DE NORMAS TÉCNICAS. NBR 7094: Máquinas elétricas girantes- Motores de Indução - Especificação. Rio de Janeiro, 2003.

AUGUSTO JÚNIOR, N.; CHABU, E. I. Motores de alto rendimento - dimensionamento e viabilidade econômica. Revista Eletricidade Moderna, São Paulo, v.336, p.109-129, 2002.

BORGES NETO, M.R.; CARVALHO, P.C.M. de. Planejamento energético rural assistido por computador. Engenharia Agrícola, Jaboticabal, v.29, n.2, p.172-184, 2009.

BRASIL. Ministério das Minas e Energia. Balanço energético nacional, 2009: Ano-base: 2008: Resultados Preliminares. Rio de Janeiro, 2009. 48 p.

CREDER, H. Instalações elétricas. Rio de Janeiro: Livros Técnicos e Científicos Editora, 2007. $517 \mathrm{p}$.

ELETROBRÁS. Disseminação de informações em eficiência energética. In: Eficiência energética aplicada para comércio e indústria. Rio de Janeiro: PROCEL, 2004. 309 p.

ELETROBRÁS. Guia operacional de motores elétricos. In: Programa de combate ao desperdício de energia elétrica - PROCEL. Rio de Janeiro: PROCEL, 2002. v.1, 161 p.

FAIZ, J.; EBRAHIMOUR, H.; PILLAY, P. Influence of unbalanced voltage supply on efficiency of three phase squirrel cage induction motor and economic analysis. Energy Conversion and Management, v.47, n.3, p.289-302, 2006.

FRIZZONE, J.A.; SILVEIRA, S. de F.R. Avaliação e custos de projetos de irrigação. In: ${ }^{\circ}$ SEMINÁRIO ENERGIA NA AGRICULTURA - CEMIG, 2000, Uberaba. Anais... s.n. v.1, p.1-87. 
LAPPONI, J.C. (Ed.) Avaliação de projetos e investimento: modelos em Excel. São Paulo: Lapponi Treinamento e Editora, 1996. 264 p.

SAIDUR, R. A review on electrical motors energy use and energy savings, renewable and sustainable energy reviews, DOI: 10.1016/j.rser.2009.10.018. 